\title{
Optical analysis of the influence of injector hole geometry on mixture formation in gasoline direct injection engines
}

\author{
Juliane Wetzel ${ }^{1}$
}

Received: 3 December 2015/Accepted: 20 April 2016/Published online: 2 May 2016

(C) Springer International Publishing Switzerland 2016

\begin{abstract}
New and increasingly strict emission standards demand a reduction in the raw emissions from today's gasoline direct injection engines. The optimisation of mixture formation by means of the injector and, in particular, the injector hole geometry offers significant potential in this respect. To achieve this, however, it is crucial to have a basic understanding of the influence of the geometry on spray breakup. To investigate this influence, a test matrix with ten different injector hole geometries was created. This included cylindrical, convergent, divergent and stepped injector holes. Different measuring techniques were used to analyse the microscopic and macroscopic spray parameters as well as the internal flow in the nozzles.
\end{abstract}

Keywords Direct injection - Injector hole geometry ·

Spark ignition engine - Mixture formation

$\begin{array}{ll}\text { Abbreviations } \\ \text { ASB } & \text { Start of injection } \\ \text { CFD } & \text { Computational fluid dynamics } \\ \text { CO } & \text { Carbon monoxide } \\ \text { GDI } & \text { Gasoline direct injection } \\ \text { HC } & \text { Hydrocarbon emissions } \\ D & \text { Injector hole diameter } \\ k \text {-factor } & \text { Unit of measure for the conicity of a nozzle } \\ 1 / D & \text { Ratio of length to diameter } \\ n & \text { Index of refraction } \\ \text { NMHC } & \text { Not methane hydrocarbon emissions } \\ p & \text { Pressure } \\ \text { PDA } & \text { Phase Doppler anemometry }\end{array}$

Juliane Wetzel

juliane.wetzel@volkswagen.de

1 Volkswagen AG, Wolfsburg, Germany

$\begin{array}{ll}S & \text { Penetration } \\ v & \text { Speed } \\ \text { VCO } & \text { Valve covered orifice } \\ \alpha & \text { Spray angle } \\ \rho & \text { Density }\end{array}$

\section{Introduction}

The main development objective for vehicles with combustion engines is to reduce fuel consumption and emissions. Attempts are being made to meet current and future emission standards by means of engine modifications, such as downsizing, extensive use of turbocharging, and direct injection in spark ignition engines, as well as variable camshaft control. In particular, the EU6 emission standard, which entered into force in September 2014, see Table 1, limits the particle count (PN, blue background) to $6 \times 10^{12}$ for the first time.

This EU6c limit, which will be increased to $\mathrm{PN}=6 \times 10^{11}$ with effect from 2017, sets new challenges, which must be confronted with the help of engine modifications. Among the main causes of particle formation are wetting of the piston and wall, wetting of the cylinder roof, including spark plugs, coking of the injector tips, local grease mixtures with $\lambda<0.5$ and the diffusive combustion of liquid fuel [2, 3].

By adapting the injector design, for example by changing the spray target or the injector hole geometry and by optimising the installation position of multi-hole injectors, it is possible to influence the microscopic and macroscopic spray parameters, such as the spray angle, penetration depth, droplet size and evaporation. In this way, it is possible to minimise the causes of increased particle emissions from within the engine. 
Table 1 European emission standard for passenger cars with gasoline engines [1]

\begin{tabular}{lllllll}
\hline Standard & NOx $(\mathrm{g} / \mathrm{km})$ & $\mathrm{CO}(\mathrm{g} / \mathrm{km})$ & $\mathrm{HC}(\mathrm{g} / \mathrm{km})$ & NMHC $(\mathrm{g} / \mathrm{km})$ & $\mathrm{PM}(\mathrm{g} / \mathrm{km})$ & $\mathrm{PN}(\# / \mathrm{km})$ \\
\hline Euro 5 & 0.06 & 1 & 0.1 & 0.068 & 5 & - \\
Euro 6b & 0.06 & 1 & 0.1 & 0.068 & 4.5 & 6.5 \\
Euro 6c & 0.06 & 1 & 0.1 & 0.068 & 6.5 & $6^{*}$ E11 \\
\hline
\end{tabular}

Injector tip coking, which is caused by fuel deposits that occur during the injection process, is a main cause of particle formation, see Fig. 1a. This is facilitated, in particular, by the current injector hole and needle seat design with stepped injector holes. These have recirculation areas in which fuel residues collect and condense. These residues further enrich the deposits (Fig. 1b), which, over time, extend into the injector holes themselves and the needle seat $[4,5]$.

Optimisation of the injector hole geometry, in particular, the omission of a stepped bore, is a promising measure to help reduce injector tip coking and thus also particle emissions. The challenge here, however, is to illustrate a combustion-optimised mixture formation process, which also avoids component wetting and offers homogeneous mixture distribution. The objective of this paper, derived from this challenge, is to provide an optical analysis of alternative injector hole geometries to evaluate the mixture formation. A further aim is to characterise the spray breakup mechanisms.

Former investigations regarding the nozzle hole geometry focussed mostly on diesel engines. For a detailed summary of the work carried out, see Gostic [7]. The very few examinations of nozzle hole geometry in gasoline direct injection engines are restricted to the length-to-diameter ratio [8, 9], the inlet shape of the nozzle hole [8] and the nozzle hole diameter [10]. Extensive studies of
Gilles-Birth [11] based on the analysis of valve covered orifice nozzles (VCO) are not used in today's gasoline direct injection (GDI) engines. Studies on the influence of the conicity and stepped geometry of the nozzle hole geometry in GDI engines are not shown in the literature currently. Also, the tests listed confined to a very limited range of measurement techniques that do not allow a holistic assessment of the mixture formation.

\section{Materials and methods}

The optical analyses were carried out using a heatable pressure chamber with a continuous flow, which depends on the adjusted pressure (Here: $0.33 \mathrm{~m} / \mathrm{s}$ ). The analyses were carried out under various engine-like operating conditions. The following evaluation is limited to atmospheric conditions $\left(p_{\mathrm{ch}}=1 \mathrm{bar} ; T_{\mathrm{ch}}=25^{\circ} \mathrm{C}\right)$ to ensure the comparability of the different measurement techniques used in this investigation. The injection pressure was provided by a linear piston pump, with the injection pressure being varied in four stages ( $p_{\text {Rail }}=50,100,150,200$ bar $)$. Iso-octane was used as the fuel for the test by reason of comparability to nozzle flow and spray simulations. Injector actuation was time-based with a defined actuation duration of $t_{i}=1 \mathrm{~ms}$. To validate the injection quantity, the flow quantity and injection rate
Fig. 1 a Diffusive flame at a coked injector tip [6]. b Cleaned and coked injector tip [4]
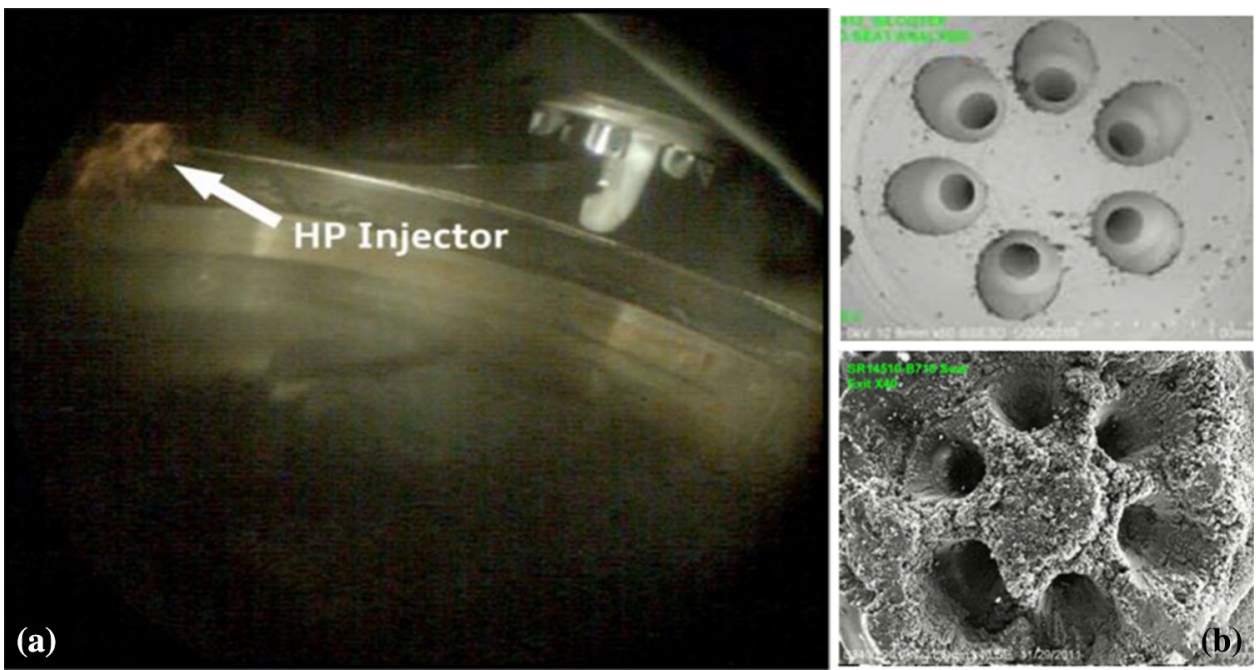
were calculated at the same time as the optical measurement.

The injectors used were based on passenger car GDI injectors, currently used in series production. These were adapted in terms of their injector hole geometry. For ease of optical accessibility, injectors with only one axially arranged injector hole were used. Cylindrical, divergent and convergent injector hole geometries were examined. An overview of the geometry characteristics of the single-hole injectors, validated with the help of computer tomography, is shown in Table 2. To reduce the effective injector hole length of variant 3 , this injector was provided with a stepped bore with a step diameter $d_{\text {Step }}$ of $500 \mu \mathrm{m}$. The step depth $L_{\text {Step }}$ results from the difference between the plate thickness of the nozzles $(1000 \mu \mathrm{m})$ and the injector hole length. All of the injectors used have a sharp inlet edge. Other characteristics in addition to the inlet diameter $D_{\mathrm{Sp}}$ are the $k$ factor and the $L / D$ ratio. The conicity was characterised using the following definition:

$k-$ Factor $=\frac{D_{\text {Inlet }}-D_{\text {Outlet }}}{10}$

A wide range of optical measuring techniques were used to characterise the mixture formation. The secondary spray breakup was analysed using a combined Mie/Schlieren measuring technique, with which it is possible to analyse both the liquid and the gaseous phases of the spray. In addition to the quantitative assessment of the images, the spray was also characterised based on the macroscopic spray parameters, which are: time-dependent penetration, the spray angle, which is calculated at two distances $(10,15 \mathrm{~mm})$ from the nozzle tip and the spray area, see also Fig. 3.

In this paper, phaseDoppler anemometry (PDA) was used to determine the chronological and local course of the sizes and velocities of spherical droplets in the spray.

The primary spray breakup was also analysed using the Mie/scattered light measuring technique, although a far field microscope was used here, making it possible to map a very small area under the nozzle exit in high optical resolution. With the help of a special evaluation method, in addition to the qualitative evaluation of the raw images, a quantitative nozzle comparison based on the droplet size, droplet diameter, droplet roundness and spray area parameters was also possible. For a detailed description of the measurement structure and the evaluation method, see [12].

Transparent nozzles were used for analysis of the nozzle internal flow. The silhouette measurement method is based on the Mie scattering of the background lighting at phase limits. Objects in the beam path can thus be detected as shadows on the photo chip. Translucent objects, with an index of refraction not equal to that of the ambient medium, appear as dark areas. The index of refraction of the acrylic glass nozzles $\left(n_{\text {Acryl }}=1491\right)$ is very similar to that of the iso-octane fuel used $\left(n_{\text {IsoOk tan }}=1391\right)$, which is why only a very slight refraction of the light occurs at the transition between the phases. By comparison, cavitation areas $\left(n_{\text {Steam }}=1000\right)$ are highlighted very clearly by the significant shadowing of the background lighting. This means it is possible to identify different phases (liquid, gaseous) inside the transparent nozzle, see Fig. 2.
Table 2 Overview of the injector hole geometries used and their geometric characteristics

\begin{tabular}{|c|c|c|c|c|c|}
\hline Short form & Geometry & Designation & $D_{\mathrm{Sp}}(\mu \mathrm{m})$ & $k$-factor $(-)$ & $1 / D(-)$ \\
\hline V1-1 & & Cylindrical & 150 & 0 & 6.67 \\
\hline V1-2 & & Cylindrical & 240 & 0 & 4.17 \\
\hline V2-1 & & Divergent & 150 & -1 & 6.67 \\
\hline V2-2 & & Divergent & 150 & -5 & 6.67 \\
\hline V2-3 & & Divergent & 150 & -10 & 6.67 \\
\hline & & & & & \\
\hline V3-1 & & Stepped & 150 & 0 & 1 \\
\hline V3-2 & & Stepped & 150 & 0 & 1.3 \\
\hline V3-3 & & Stepped & 150 & 0 & 2 \\
\hline V4-1 & & Convergent & 150 & 1 & 6.67 \\
\hline V4-2 & & Convergent & 150 & 5 & 6.67 \\
\hline
\end{tabular}




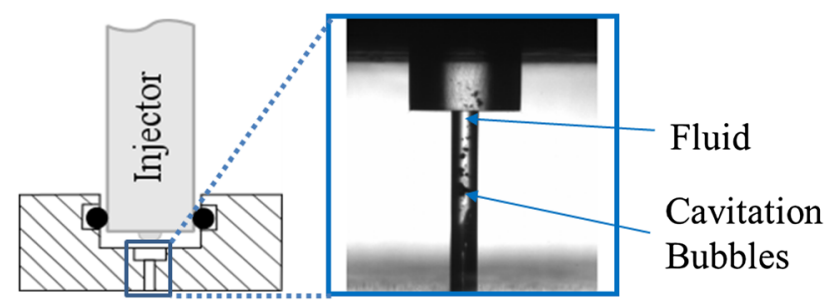

Fig. 2 Measurement setup and a typical result of the silhouette method at transparent nozzles

\section{Results and discussion}

\subsection{Macroscopic spray parameters}

A typical image sequence of the liquid fuel spray for nozzle V1-1 is shown in Fig. 3. For each time interval, the penetration $S$, the spray angle $\alpha$ at two distances from the nozzle tip (10 and $15 \mathrm{~mm}$ ) and the spray area were determined. Figure 3 compares the penetration as a mean value of ten injection processes with the injector hole geometry being tested at 50 and 200 bar fuel pressure.

Essentially, Fig. 4 shows us that an increase in the injection pressure results in a larger rise in the penetration curve and thus a higher speed of the spray front. The spray speed thus corresponds to the Bernoulli speed (calculated by $v_{\text {Bernoulli }}=\sqrt{\frac{2}{\rho}\left(p_{\text {Rail }}-p_{\text {Ambient }}\right)}$ in the injector hole, which is primarily determined by the pressure difference between the injector hole inlet and the injector hole exit. Also, we can see that the trends between the nozzle hole geometries at 50 bar fuel pressure are amplified at 200 bar fuel pressure.

Looking at the cylindrical geometries (V1-1 and V1-2), see Fig. 4 above, we can see that the smaller injector hole diameter results in a larger rise in the penetration curve. This is due in part to the self-adjusting pressure in the blind hole. The larger injector hole diameter results in an amplified pressure drop and, thus, a smaller pressure difference between the injector hole inlet and exit. According to Bernoulli, this results in a reduced fuel speed. On the other hand, the result can be explained by the spray angle or the spray area. Since the spray angle, which is shown in Fig. 5 as a mean value over the injection period, and the spray area, see Fig. 6, also have significantly larger values, the flow resistance of the spray against the ambient medium is increased. This results in a reduction in the spray speed.

The penetrations of the divergent geometries (V2-X), as shown in Fig. 4, are essentially the same as the penetrations of the cylindrical geometry (V1-1). However, there is no clear dependence of the penetration on the $k$ factor. The smallest divergence also has the smallest penetration or penetration speed. The middle $k$-factor, on the other hand, results in the highest spray speed and largest penetration depth of all divergent nozzles. With further increasing divergence (V2-3), the penetration reduces again. Similar behaviour can be observed in the spray angle (Fig. 5). At both distances from the nozzle tip, the middle divergence has the smallest spray angle. The spray angle is larger with both the small and the large $k$-factor. The causes for this cannot be explained clearly based on the previously available measured data. One possibility is the flow behaviour within the injector hole, which is highly dependent on the profile of the flowcontrolling geometry. It is supposed that excessive divergence causes the flow to separate from the walls, which significantly influences the flow parameters. This assumption is supported by the fact that the vertex of the spray angle is the centre point of the injector hole exit. When the flow lies flat against the walls, then, due to the enlarged exit diameter, there must already be an increased spray angle in variant V2-3 in particular. However, this
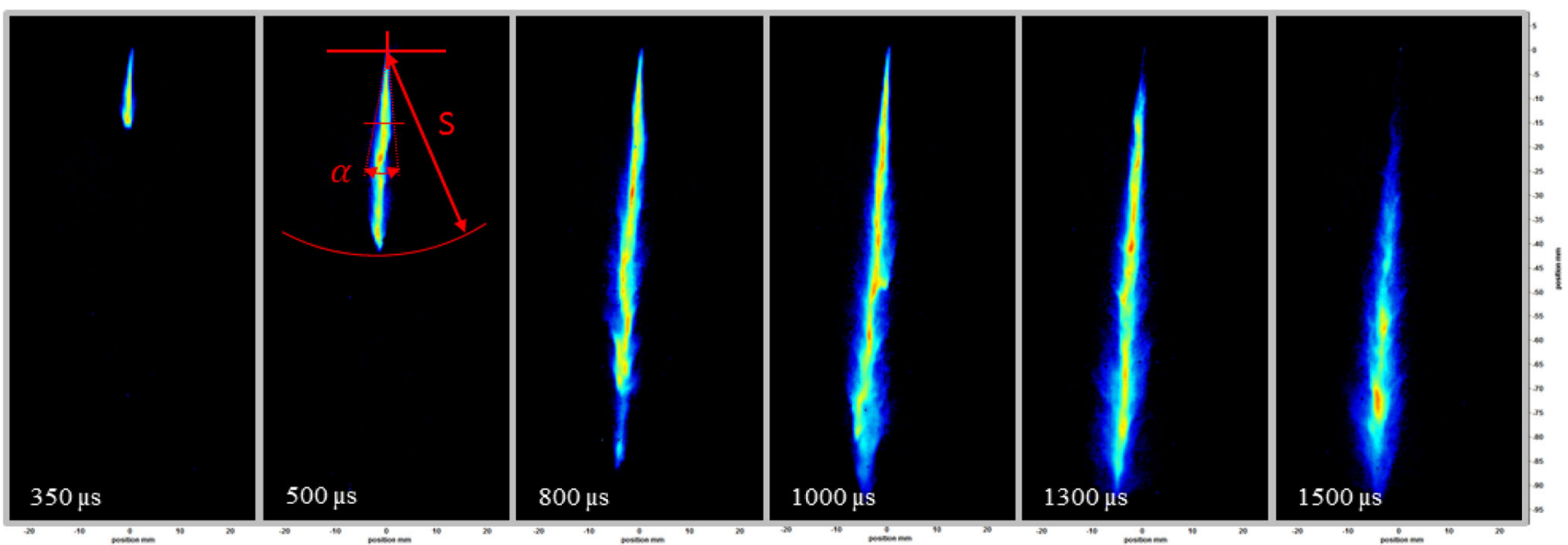

Fig. 3 Spray images of the V1-1 nozzle in false-colour representation, $p_{\text {Rail }}=200 \mathrm{bar}, t_{i}=1 \mathrm{~ms}$ for different times after the start of injection 

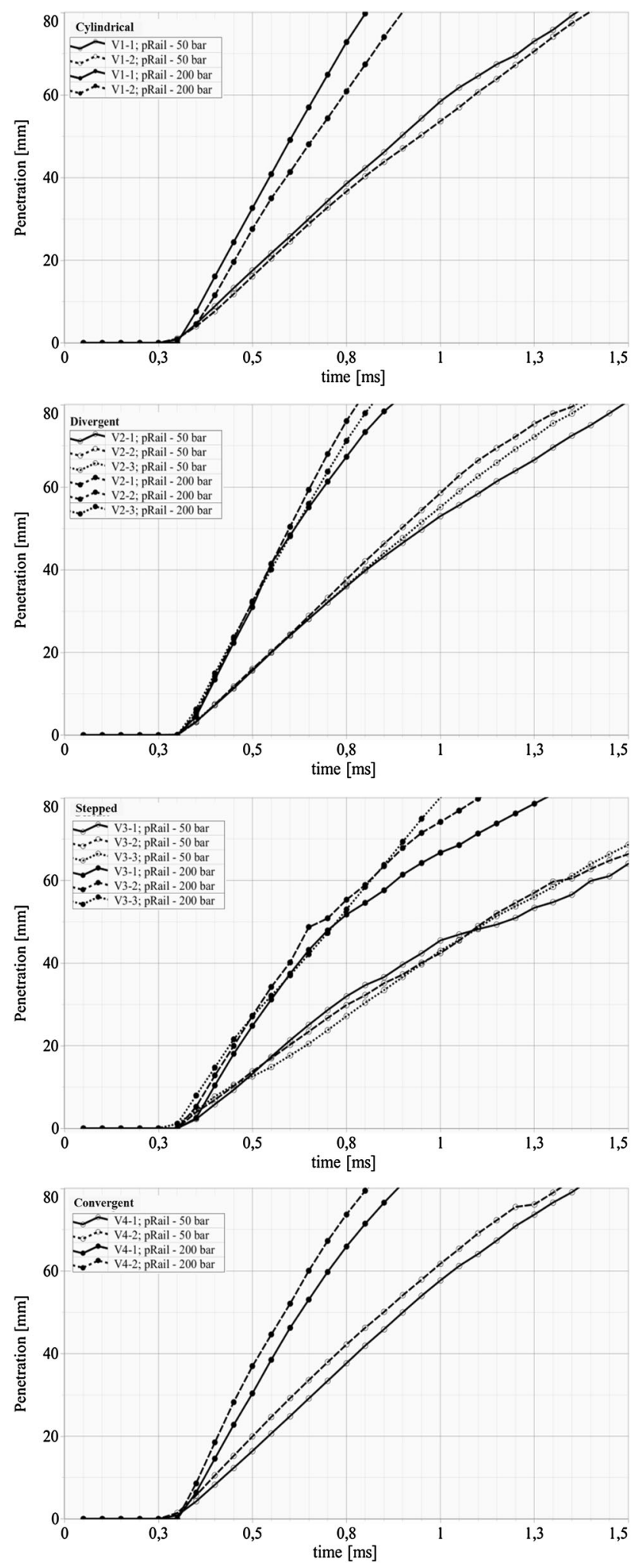

Fig. 4 Penetration curve of the different nozzle geometries at fuel pressures of 50 and 200 bar cannot be discerned and thus requires further investigation.

In principle, it can be ascertained that the stepped injector holes have the smallest penetration (Fig. 4) and the largest spray angle (Fig. 5) of all geometry variants. As the effect of the penetration reduction prevails, however, the stepped injector hole geometries have the smallest spray area of all nozzle geometries. Furthermore, it can be ascertained that the stepped injector variants have a very unsteady penetration curve. There are overlaps between the individual curves. Thus, geometry V3-1, with the shortest injector hole, has the smallest penetration shortly after the injector is opened. As the spray front continues, this geometry experiences a lesser deceleration, which results in an increased penetration. The maximum penetration of the nozzle, however, is significantly lower than the penetration depths of the longer injector hole variants. As the injector hole length increases (V3-2 and V3-3), the penetration speed also increases, and an approximation to a linear penetration curve occurs. This characteristic is also described in the references [13]. At the same time, there is a reduction in the average spray angle, whose dependency on the injector hole length is described in a differentiated manner in the reference material $[14,15]$. The turbulence in the injector hole is considered to be the cause of the increase in penetration and the reduction in the spray angle as the injector hole length increases. An increase in turbulence is caused, in particular, by cavitation, which occurs at the sharp-edged injector hole inlet. When the injector hole length is short, there is no time for flow calming, with the result that there are increased radial and reduced axial speed components at the injector hole exit with small 1/ $D$ ratios, which bring about the described phenomena. However, the reduced effective length of the injector holes also results in reduced friction losses at the injector hole walls at the same time. A combination of these mechanisms can result in the described effects of the overlapping penetration curves with different $1 / D$ ratios.

The convergent injector hole variants (V4-X) have penetration curves which are very similar to those of the cylindrical and divergent variants. Both at 50 and at 200 bar fuel pressure, there is a clear increase in the penetration speed of nozzle V4-2. The convergence results in an acceleration of the fuel and, thus, an increased exit speed and increased penetration depth. Figure 5 also shows that the spray angle is dependent on the conicity. As the $k$ factor rises, the spray angle falls by approximately $20 \%$. This is largely due to the constriction of the flow at the injector hole exit. The slightly convergent nozzle, in comparison with the other geometries, has a significantly 
Fig. 5 Average spray angle of the different nozzle geometries at 50 bar fuel pressure

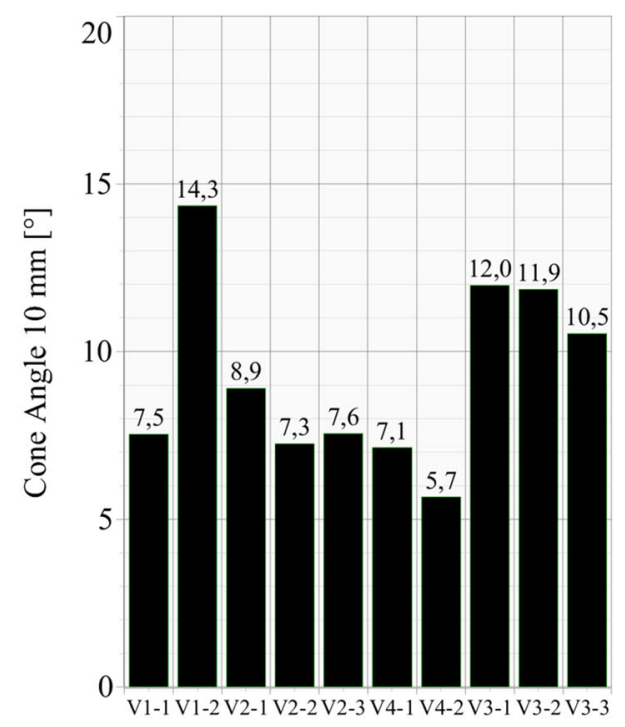

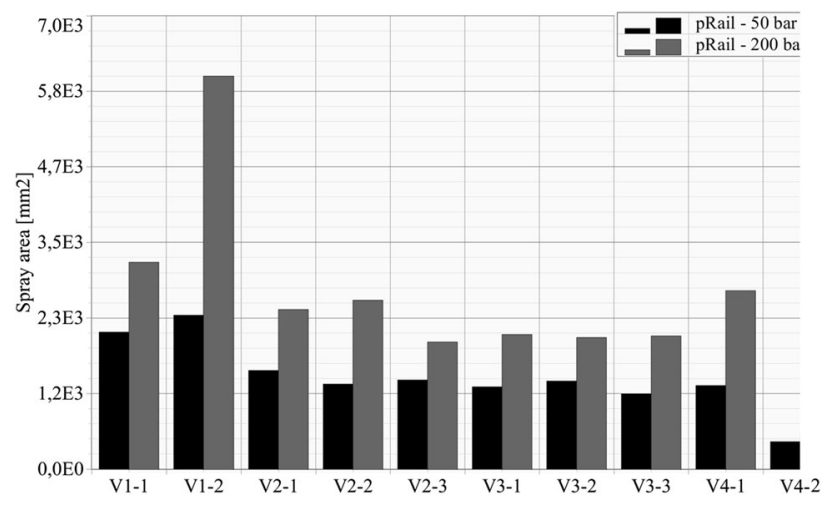

Fig. 6 Spray area of the liquid phase of all nozzle geometries at 50 and 200 bar fuel pressure

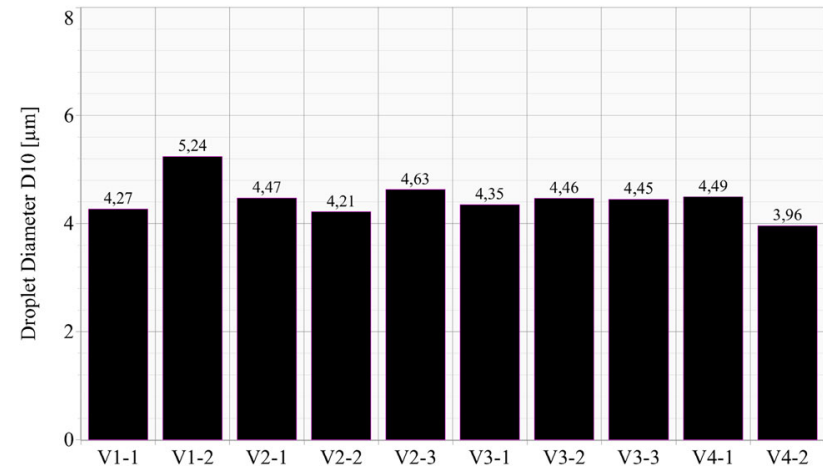

Fig. 7 Average droplet diameter D10 at 200 bar fuel pressure

larger spray area at 200 bar injection pressure in particular. This can be seen by the good breakup of the fuel spray. The highly convergent nozzle, on the other hand, has a significantly smaller spray area. It should be noted that, due to the significant constriction, the flow is reduced and, thus, variant V4-2 injects a significantly smaller quantity of fuel over a constant actuation duration of $1 \mathrm{~ms}$. It is this reduced spray density that causes the observed smaller spray area. It does not allow any conclusions to be drawn about the atomisation quality.

The droplet diameter, which was determined $30 \mathrm{~mm}$ below the injector tip, also differs depending on the nozzle geometry. Figure 7 compares the average arithmetic droplet diameter for the different variants. Nozzle V1-2, which has the largest basic hole diameter, also has the largest droplet diameter. This is in agreement with the references, which specifies the injector hole diameter as the main criterion for the droplet size [16-19]. This is also the cause of the smallest droplet diameter of nozzle V4-2, which has the smallest exit diameter of all variants. However, this theory cannot be applied to the nozzles with a divergent geometry. It can be ascertained that it is not the exit diameter that is crucial for the droplet diameter, but rather the smallest, restricting diameter. In the case of the divergent injector holes, this is the inlet diameter. The differences between the remaining geometries are very minor. However, very similar trends can be identified, as with the penetration and spray angle analyses. Thus, the injector hole with medium divergence has the smallest average droplet diameter both of the divergent nozzles and of the nozzles with an identically small injector hole diameter. The droplet diameter and spray area of nozzle V2-2 indicate an optimised spray breakup due to the injector hole geometry.

\subsection{Microscopic spray parameters}

The spray breakup that occurs near the nozzle, called primary breakup, is crucial for the secondary spray breakup, 

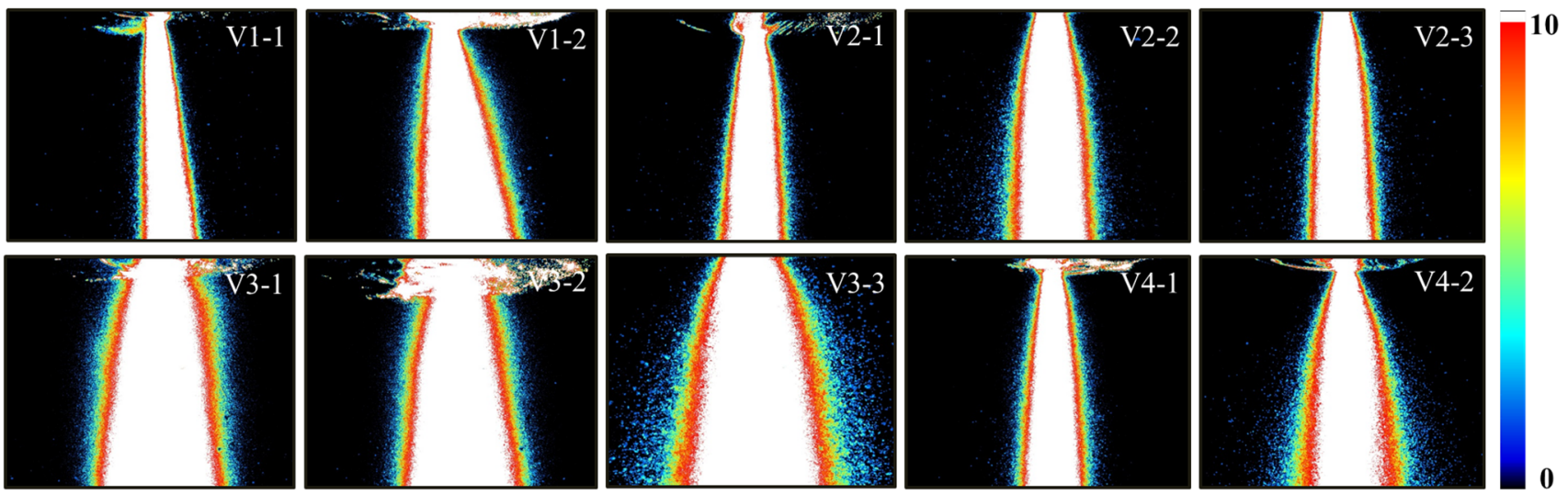

Fig. 8 Primary spray development of the nozzle variants at 100 bar injection pressure, total of ten images taken 0.8 ms after the start of injection

which occurs away from the nozzle. Primary spray breakup is the breakdown of a liquid, coherent spray into first ligaments and large droplets. The main influences on this are aerodynamic interactions, turbulence, profile relaxation and cavitation [20]. Using a far field microscope, it is possible to visualise this area very close to the nozzle tip (approx. $3.5 \times 2.5 \mathrm{~mm}$ ). Below, a selection of measurement results are presented to assess the influence of the injector hole geometry on the primary spray breakup.

Figure 8 compares the images of one injection time (0.8 $\mathrm{ms}$ after start of injection) for all injectors. To take into consideration the cyclic fluctuations, the binarised images of ten injection processes were summated. In the images, it can be seen that both an increased nozzle hole diameter (V1-2) and a reduced injector hole length (V3-X) significantly increase the spray area. This is due, on the one hand, to the increased mass flow (nozzle V1-2) and also to the increased turbulence and, thus, the amplified spray development (nozzle V3-X). As, however, nozzle V3-3, with the largest 1/ $D$ ratio, has the strongest spray development of the stepped nozzles, the injector hole length cannot be the only cause. It can be assumed that both the step diameter and the ratio of step depth to step diameter have a significant influence on the spray development energy.

The slightly convergent nozzle (V4-1) has significantly smaller cyclic deviations over the entire injection period, which is due to the familiar effects from diesel injection technology: Due to convergence, the air flow pressure in the injector hole is increased, the flow is calmed, and cavitation is limited. This is contrasted with the increase in spray area and the cyclic fluctuations in nozzle V4-2 with a $k$-factor of +5 . Due to the significant convergence, the exit speed increases, which is a main criterion for the spray development. The images show that the spray angle is significantly larger. A further cause could be the increase in radial speed components due to a cross-flow in the injector hole.
Quantitative assessment of the spray breakup near the nozzle is carried out based on Fig. 9. The time curve of the spray area and droplet size spray characteristics of all nozzle variants being examined at 100 bar injection pressure are shown. In the diagrams, the injection phases are clearly visible. The first droplets are detected approximately $0.23 \mathrm{~ms}$ after the start of injection. It must be pointed out that the droplets that are detected before this time, are caused by background noise or reflections and, due to the very low number of detected particles in the evaluation of the droplet diameter, are over-interpreted. This results in a sharp increase in the droplet size and spray area. This phase of the maximum droplet size and spray area is caused by needle seat throttling and the associated turbulence and cavitation within the injector hole. The phenomenon of needle seat throttling and the associated effects are already described in detail in the references [21]. After complete opening of the needle, both the spray area and the droplet diameter assume constant values. The closing phase (from $1.1 \mathrm{~ms}$ ) is also identified by slight exaggerations in the curves for the spray area and a sharp increase in the droplet size. The reason for this is the pulse loss of the spray, brought about by needle seat throttling and the falling nozzle flow. Large ligaments escape through the injector hole, which have a slight circularity. Due to the low fuel speed, the forces acting on the droplet, and the aerodynamic interactions with the ambient air, are low.

At this stage, only the constant needle opening phase is used for further characterisation of the spray development and for comparison of the individual nozzle variants $(t=0,6 \ldots 1,0 \mathrm{~ms})$. It must again be noted that the droplet diameter is generally very small $(4-8 \mu \mathrm{m})$. This is due to the evaluation method, which can only detect droplets that are clearly delimited from the background. Large droplets and ligaments, which occur within the dense fuel mist, cannot be detected and, thus, reduce the measured droplet diameter in comparison with the actual droplet diameter. 

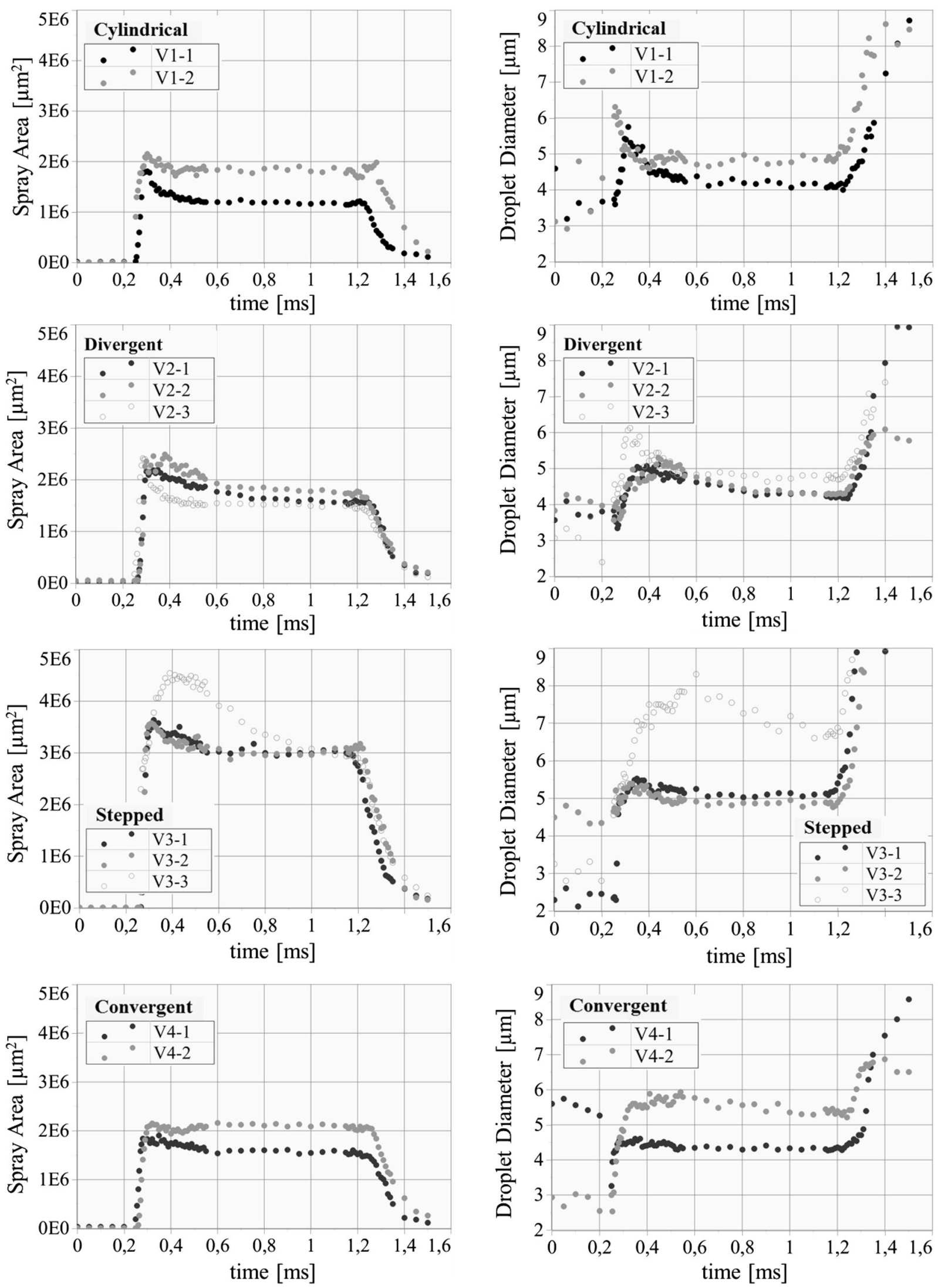

Fig. 9 Comparison of the time curve of the droplet size and spray area in the area near the nozzle for all examined injectors at 100 bar injection pressure 


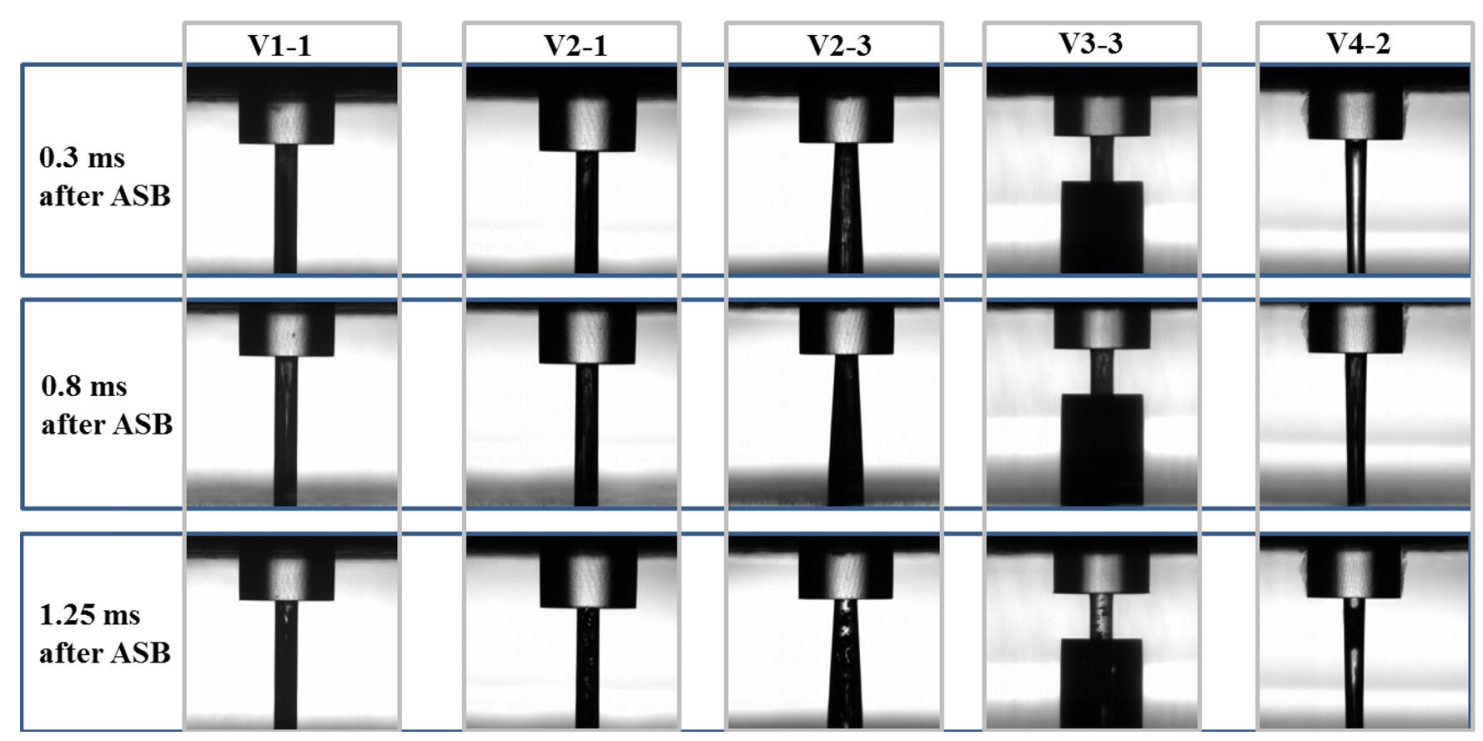

Fig. 10 Silhouettes of the internal nozzle flow of selected geometries at 100 bar injection pressure

Figure 9 also shows that the stepped nozzle V3-3, which appeared very striking in the spray images, also stands out from the other nozzles in this quantitative evaluation. It has a significantly larger spray area as well as a significantly larger droplet diameter. The spray area of the other stepped nozzles V3-1 and V3-2 also stands out clearly from the remaining nozzles, but these nozzles have significantly smaller droplets than nozzle V3-3. This confirms the assumption that, in addition to the $1 / D$ ratio, the stepped geometry plays a key role in the spray breakup.

A comparison of the divergent nozzles shows that, as with the spray images, there is no discernible trend between the variants. The spray area for variant V2-3 is minimal. Nozzle V2-2 has the maximum spray area, and nozzle V2-1, with the lowest divergence, lies between these two variants. This confirms the assumption that the breakup mechanisms do not have a linear dependence on the divergence, but that there is a return point, which is associated with occurrences of separation from the injector hole wall. The droplet diameters barely differ in the divergent nozzles in contrast.

The cylindrical injector hole (V1-1) has the smallest spray area of all nozzles, although the droplet diameter is at the same level as in the divergent variants. As the nozzle diameter increases (V1-2), the spray area and droplet diameter also increase. This was also discernible in the macroscopic spray parameters.

The convergent nozzles are characterised by widely varying droplet diameters and spray areas. Nozzle V4-2, at approximately $5.8 \mu \mathrm{m}$, has the second largest average droplet diameter of all nozzles, while variant V4-1, at $4.2 \mu \mathrm{m}$, has the smallest average droplet diameter. A similar result can be obtained analysing the spray area, as the nozzle with the large $k$-factor has a significantly amplified spray area. This allows us to conclude that convergence causes strong breakup mechanisms. It has to be noticed that there is a contradictory tendency at the droplet diameter of the convergent nozzle measured by PDA and the far field microscope. It has to be determined that the droplet diameter near the nozzle cannot be directly linked to the droplet diameter in the far field. A strong spray breakup near the nozzle exit leading to big droplets can have a positive effect on the secondary breakup mechanism.

\subsection{Transparent nozzles}

Using transparent nozzles, it was possible to characterise the flow inside the nozzles. Figure 10 compares the flow inside the nozzles at selected times (needle opening, stationary phase and needle closing) and the injector hole geometries at 100 bar injection pressure. It is clear that, with all geometries, there are shadows in the injector hole i.e., cavitation. As expected in accordance with the spray analyses, we see that when the needle is opened, needle seat throttling occurs and, as a result of this, cavitation occurs in the blind hole, which then extends into the entire injector hole as film cavitation. Due to the integrative measuring method of the silhouette technique, no conclusion can be drawn about the thickness of the cavitation film based on the measurement results. Further tests are required. However, it must be noted that cavitation occurs even with geometry V4-2. In the field of diesel injection technology, the convergent injector hole geometry is successfully used to avoid cavitation. It is clear that the strong convergence and, thus, the increase in air flow pressure are 
not sufficient to prevent cavitation under the conditions present here. The reason for this could lie in the fluid properties. Iso-octane $\left(p_{D}=0.0439\right.$ bar $)$ has a significantly higher vapour pressure than diesel (ca. $p_{D}=0.0005$ bar). Also, the sharp inlet edge is rounded off in diesel injectors. With the nozzles used here, a significant deflection occurs at the area of the sharp inlet edge, and a sudden constriction of the fluid flow occurs, which promotes the occurrence of cavitation even at the lowest of injection pressures ( $p_{\text {Rail }}<50$ bar $)$.

\section{Summary}

To meet future emission standards, optimisation of spray development is crucial. A basic understanding of the primary and secondary breakup processes and the geometric influences of the nozzle geometry on these processes are, however, a requirement in designing the injectors and optimising mixture formation.

This paper forms the basis for acquiring a fundamental understanding of the geometric influences of injector holes on mixture formation in gasoline direct injection engines. Using a wide range of measuring techniques, it was possible to carry out optical analysis of the flow inside the nozzles, primary spray development and secondary spray breakup.

It was possible to show that the nozzle geometry is fundamentally important, in particular, in terms of the primary spray breakup. The droplet diameter is crucially dependent on the minimum injector hole diameter. There is no linear connection between the $k$-factor of the divergent nozzles and the spray breakup. Thus, with the largest $k$ factors, the flow separates from the walls, which has a negative influence on the spray development. Therefore, an optimal divergence is, in this investigation, a $k$-factor of -5 . Different boundary conditions and alternative designs can lead to different optimal $k$-factors. As the $1 / D$ ratio falls, the turbulence in the spray increases and the spray development is amplified. In addition to the $1 / D$ ratio, however, the ratio of step depth to step diameter also significantly influences the spray breakup in stepped injector holes. With convergent injector holes, the relative speed between the fuel and the ambient medium can be increased, and the flow can be given a radial speed component, with the result of amplified spray development with very small droplet sizes. However, cavitation cannot be avoided through the use of convergent nozzles. Due to sharp intake edges and the fluid properties, cavitation occurs even with the smallest of fuel pressures. Adapting the inlet hole geometry offers the potential of making a positive influence on mixture formation and, thus, helping to reduce raw emissions.

\section{References}

1. Euro 5/6 Standards: Regulation 715/2007 and several comitology regulations. http://www.dieselnet.com/standards/eu/ld.php (2009/ 2014)

2. Steimle, F., Kulzer, A., Richter, H., Schwarzenthal, D., Romberg, C.: Systematic analysis and particle emission reduction of homogeneous direct injection SI engines. SAE technical paper 2013-01-0248. (2013). doi:10.4271/2013-01-0248

3. Winkelhofer, E., Fuchs, H., Hirsch, A., Kapus, P.: Stabilität der Partikelemissionen von GDI Brennverfahren-Diagnose im Motorbetrieb", Diesel-und Benzindirekteinspritzung (7th congress), 279-287, Berlin, ISBN 978-3-8169-3180-5 (2013)

4. Imoehl, W., Gestri, L., Maragliulo, M., Del-Frate, L., Klepatsch, M., Wildeson, R.: A DOE approach to engine deposit testing used to optimize the design of a gasoline direct injector seat and orifice. SAE Int. J. Fuels Lubr. (2012). doi:10.4271/2012-01-1642

5. Birgel, A., Ladommatos, N., Aleiferis, P., Milovanovic, N., Lacey, P., Richards, P.: Investigations on deposit formation in the holes of diesel injector nozzles. SAE 2011-01-1924 (2011)

6. Wurms, R., Budack, R., Grigo, M., Mendl, G., Heiduk, T., Knirsch, S.: The new Audi 2.01 engine with innovative Rightsizing - a further Milestone in TFSI Technology. 36th International Vienna Motor Symposium, Vienna (2015)

7. Gostic, I.: Experimentelle Studie des Strahlzerfalls in Abhängigkeit von der Düsengeometrie. Dissertation, Universität Erlangen-Nürnberg, Erlangen (2011)

8. Schulz, F., Schmidt, J.: The influence of Gasoline nozzle geometry on spray formation and piston wetting. Fista (2014)

9. Moulai, M., Grover, R., Parrish, S., Schmidt, D.: Internal and near-nozzle flow in a multi-hole gasoline injector under flashing and non-flashing conditions. SAE technical paper 2015-01-0944 (2015)

10. Abe, M., Hideharu, E., Masahiro, S., Ishikawa, T.: Spray atomization study on multi-hole nozzle for direct injection gasoline engines. SAE technical paper 2013-01-1596 (2013)

11. Gilles-Birth, A.I.: Optische Untersuchung der Düseninnenströmung von Sitzlochinjektoren für die Benzindirekteinspritzung. Dissertation, Universität Karlsruhe, Karlsruhe (2008)

12. Wetzel, J., Henn, M., Gotthardt, M., Rottengruber, H.: Experimental investigation of the primary spray development of GDI injectors for different nozzle geometries. SAE technical paper 2015-01-0911. doi:10.4271/2015-01-0911 (2015)

13. Varde, K.S., Popa, D.M.: Diesel fuel spray penetration at high injection pressures. w: SAE technical paper (830448) (1983)

14. Reitz, R.D., Bracco, F.B.: On the dependence of spray angle and other spray parameters on nozzle design and operating conditions. w: SAE Technical Paper (790494) (1979)

15. Wu, K.J., Su, C.C., Bracco, F.V., Steinberger, R.L., Santavicca, D.A.: Measurement of spray angle of atomizing jets. w: Transactions of the ASME (1983)

16. Kamimoto, T., Matsuoka, S.: Prediction of spray evaporation in reciprocating engines. w: SAE Technical Paper (770413) (1977)

17. Arrègle, J., Pastor, J.V., Ruiz, S.: The influence of injection parameters on diesel spray characteristics. w: SAE Technical Paper (1999-01-0200) (1999)

18. Varde, K.S., Popa, D.M., Varde, L.K.: Spray angle and atomization in diesel sprays. w: SAE Technical Paper (841055) (1984) 
19. Hiroyasu, H., Arai, M., Tabata, M.: Empirical equations for the Sauter mean diameter of a diesel spray. In: SAE technical paper 890464 (1989)

20. Fath, A.: Charakterisierung des Strahlaufbruch-Prozesses bei der instationären Druckzerstäubung. Dissertation, University of Erlangen-Nuremberg (1997)
21. Papoulias, D., Giannadakis, E., Mitroglou, N., Gavaises, M.: Cavitation in fuel injection systems for spray-guided direct injection gasoline engines. SAE technical paper 2007-01-1418 (2007) 